\title{
Croissance et comportement alimentaire d'alevins de Brochet (Esox lucitus, L.) élevés en cages dans le réservoir de Pareloup
}

\author{
C. Lejolivet ${ }^{1}$ \\ F Daubal
}

Mots clés : alevins de Brochet, cages, zooplancton, croissance. stratégies d'alimentation.

Des alevins de Brochet (Esox lucius, L.) ont été élevés en cages immergées dans le lac de Pareloup, selon 4 rations de zooplancton et 2 mises en charge.

La croissance, le rythme de prise alimentaire, le régime alimentaire et la sélection des proies, ont été étudiés pendant 16 jours de leur phase planctonophage.

Quelles que soient leur taille et leur ration, les alevins sélectionnent activement les proies de taille comprise entre 400 et $800 \mu$. Bosmina longirostris représente 51 à $80 \%$ des proies ingérées.

Pour 16 jours d'élevage, la ration optimale est de 17 grammes de plancton par cage, pour une mise en charge initiale de 600 alevins $/ \mathrm{m}^{3}$.

Growth and feeding behaviour of Ptke (Exos lucius, L.) fry reared in cages in Pareloup reservoir

Keywords : Pike fry, cages, zooplankton, growth, feeding strategies.

Rearing of fry pike (Esox lucius, L.) at two densities, has been performed in Pareloup Lake, by feeding on 4 zooplankton intakes.

Fish growth, diel feeding activity, diet and food selection, are studied for 16 days during the planktivorous stage.

The fry shows actively selection on $\mathbf{4 0 0 - 8 0 0 ~} \mu$ zooplankton with regardless of fish size and ration. Bosmina longirostris represents 51 to $80 \%$ of ingested preys.

For the 16 rearing days, the optimal food intake is of 17 grams plankton by cage with an initial density of $600 \mathrm{fry} / \mathrm{m}^{3}$.

\section{Introduction}

La production contrôlée d'alevins de Brochet en étang ou en cages illuminées a fait l'objet de nombreuses études (Fago 1977, Souchon 1980, Bry \& Gillet 1980, Jager 1982, Boutry 1983, Zaugg \& Pedroli 1984). Les résultats obtenus restent très globaux et concernent, le plus souvent, des bilans obtenus à l'issue de la période de planctonophagie, du stade " fingerling ", ou du stade " estivaux ". Parfois les régimes alimentaires sont décrits pour les différents écophases de ces poissons (Hunt \& Carbine 1950,

1. Laboratoire d'Ichtyologie appliquée. Ecole Nationale Superieure Agronomique, 145 avenue de Muret, 31076 Toulouse Cédex (France).
Beyerle 1968, Chodorowska \& Chodorowski 1969. 1975, Applegate 1981).

Peu de travaux sont consacrés à l'étude des processus nutritionnels pendant la phase planctonophage, phase que tous les auteurs s'accordent à désigner comme la plus critique (Gulland 1965, Chenoweth 1970).

La disponibilité d'une nourriture adéquate pendant cette phase conditionne la croissance, la survie et le passage plus ou moins précoce au régime ichtyophage.

L'étude, concernant l'alevin de Brochet, du réservoir de Pareloup a été axée sur cette période dont le déroulement est le plus souvent influencé par les conditions climatiques rigoureuses et les contraintes physiques (marnages). 
Le protocole mis en auvre vise à acquérir pour cet te période, des indications sur la croissance journalière et le comportement alimentaire el à définir une ration optimale de plancton par cage.

Le réservoir de Pareloup, d'une superficie de 1350 ha, est situé à 805 mètres d'altitude sur le plateau du Lévezou, dans le département de l'Aveyron. C'est un lac oligotrophe à tendance mésotrophe. La température moyenne annuelle est de $11^{\circ} \mathrm{C}$. Les premières données hydrobiologiques recueillies, remontent à 1981 (Dauba 1983).

Le peuplement piscicole est composé de 14 espèces parmi lesquelles dominent le Gardon, la Perche et le Brochet.

\section{Matériel et méthodes}

Les alevins ont été obtenus en mai 1984 par reproduction artificielle effectuée sur le site à partir de géniteurs du réservoir, capturés aux filets maillants. Les brochetons ont été répartis en 4 cages de $1 \mathrm{~m}^{3}$, suspendues à un ponton ancré à $30 \mathrm{~m}$ du rivage (cages réalisées selon le modèle de Jager, communication personnelle). Le tissu synthétique utilisé, d'une maille de 200 microns, ne permet tait que les échanges d'eau.

Le plancton récupéré par filtration à l'aide d'une pompe immergée à 2 mètres au-dessous du ponton, a été distribué chaque matin à $9 \mathrm{~h}$.

La période d'étude est de 16 jours (4 jours d'acclimatation et passage au régime ichtyophage déduits).

Les mises en charges et les rations totales de plancton distribuées par cage sont les suivantes:

\begin{tabular}{lcc}
\hline Cage & Mise en charge & Ration \\
\hline cage 1 & 600 & 24900 proies \\
cage 2 & 600 & 9 grammes $(\mathrm{n})$ \\
& & 470000 proies \\
cage 3 & 600 & 17 grammes $(2 \mathrm{n})$ \\
& & 912000 proies \\
cage 4 & 300 & 33 grammes $(4 \mathrm{n})$ \\
& & 1732000 proies \\
& & 63 grammes $(>4 \mathrm{n})$
\end{tabular}

Pendant la durée de l'expérience, la température a varié de 11 à $15^{\circ} \mathrm{C}$; la concentration en oxygène

s'est maintenue à saturation; le pH est resté inférieur à la neutralité.

Des alevins ont été prélevés régulièrement au cours de cet te période pour l'étude de la croissance et des habitudes alimentaires. Les échantillons de plancton distribué et les alevins sacrifiés ont été conservés dans de l'alcool glycériné à $10 \%$. Les alevins ont été mesurés à $0.5 \mathrm{~mm}$ près et pesés à $0.1 \mathrm{mg}$ près. La largeur de la bouche a été mesurée à $0.1 \mathrm{~mm}$ près.

La détermination, le comptage et la taille du zooplancton des rations et des contenus digestifs ont été effectués sous binoculaire au grossissement 30 et sous microscope au grossissement 40 . La mesure des Cladocères ne tient pas compte de l'épine caudale, la longueur totale des Copépodes n'inclue pas les soies furcales.

Le poids des contenus stomacaux, en milligram. mes, ont été calculés à partir du poids moyen des proies zooplanctoniques $(0.038 \mathrm{mg})$ et ramenés au poids moyen des alevins. Ce rapport représentc l'état de réplétion de l'estomac (CR).

Les croissances linéaire et pondérale ont été ajus. tées selon le modèle exponentiel suivant:

$\mathbf{L}=\mathbf{a} \times \operatorname{Exp}(\mathrm{bt})$

$\mathrm{L}$ : longueur totale en $\mathrm{mm}$

$t$ : jours d'experience

Les relations poids-longueur ont été calculées selon la formule suivante :

$$
\begin{array}{ll}
W=A \times L^{B} & W: \text { poids frais en } \mathrm{mg} \\
& \mathrm{L}: \text { longueur totale en } \mathrm{mm}
\end{array}
$$

L'accroissement moyen journalier en longueur i $\Delta$ $\mathbf{L m m} / \mathbf{j}$ ) et le taux de croissance linéaire correspondant $(\mathrm{LL} \% \mathrm{~mm} / \mathrm{j})$ ont été calculés à l'issue des 16 jours d'élevage.

Le coefficient de croissance pondérale total (G) a été calculé pour chaque cas expérimental, comme suit :

$\mathbf{G}=-\log (\mathrm{Wf} / \mathrm{Wi})$

Wf : poids en fin d'expérience

Wi : poids initial

Lc comportement alimentaire des brochetons a été étudié selon les critères suivants :

- la taille de l'alevin (classes de $4 \mathrm{~mm}$, de 12 à $24 \mathrm{~mm}$ )

- la taille totale distribuée par cage (n, $2 n, 4 n$, $>4 n$ ).

- la mise en charge initiale ( 300 et 600 alevins). 
Différents points ont été examinés :

- le rythme de prise a limentaire sur un cycle de $24 \mathrm{~h}$, par prélèvément d'alevins toutes les 3 heures el suivi du coefficient de réplétion (CR);

- le nombre moyen de proies par tube digeslif, le taux de cannibalisme ;

- la composition du régime alimentaire par l'étude des fréquences d'occurence des différentes proies, sur l'ensemble de la période étudiée ;

- la relation entre la longueur des proies et la taille de l'alevin. Pour chaque poisson, la longueur moyenne des proies ingérées a été calculée; la longueur de la proie la plus grande et celle de la proic la plus petite ont été notées. Les moyennes de ces 3 paramètres (Lmoy., Lmax., Lmin.) ont été calculées pour chacune des classes de taille d'alevins.

- la sélection des proies a été étudiée à partir des contenus digestifs des alevins prélevés au cours du cycle de $24 \mathrm{~h}$. L'indice d'électivité d'Ivlev a été calculé pour les 5 catégories de proies retenues, cha. cune d'elle répartie en classes de taille de 400 microns, selon la formule suivante:

$$
\mathrm{E}=(\mathrm{ri} \cdot \mathrm{pi}) /(\mathrm{ri}+\mathrm{pi})
$$

ri : proportion de la proie $\mathrm{i}$ dans le tube digestif $\mathrm{pi}$ : proportion de la proie $\mathbf{i}$ dans la nourriture

$E$ varie de -1 à +1 . La valeur -1 exprime un évitement total de la proie; la valeur nulle, une sélection passive et la valeur +1 , une sélection totale de cette proie.

\section{Résultats et discussion}

\subsection{Croissance}

Seuls les brochetons planctonophages au moment des prélèvements ont été pris en considération pour la représentation graphique de la croissance (Tableau I, fig. 1). Les valeurs des paramètres de croissance tenant compte des poissons ichtyophages sont données à titre indicatif.

A l'issue des 16 jours d'expérience les longueurs sont comprises entre $17 \mathrm{~mm}$ (cage 4) et $20 \mathrm{~mm}$ (cage 2) ; les poids, entre $27 \mathrm{mg}$ (cage 4) et $50 \mathrm{mg}$ (cage 2).

La meilleure croissance est observée pour la cage 2 , ayant reçu une ration totale de 17 grammes de plancton. Ce sont pour des conditions de faible mise en charge et/ou de forte ration que les plus faibles croissances ont été observées.
Tableau I. Paramètres des courbes de crojssance linéaire et pondérale et des relations poids-longueur.

$\mathrm{L}$ : longueur totale (mm) W: poids frais total (mg)

$t$ : jours d'expérience

- alevins planctonophages

- alevirs ichryophages compris

\begin{tabular}{|c|c|c|c|c|}
\hline & Cage 1 & Cage 2 & Cage 3 & Cage 4 \\
\hline a & $\begin{array}{l}11.7145 \\
11.4159\end{array}$ & $\begin{array}{l}11.4474 \\
10.5538\end{array}$ & $\begin{array}{l}11.0067 \\
10.3975\end{array}$ & $\begin{array}{l}11.7262 \\
10.3518\end{array}$ \\
\hline D & 0.0333 & $\begin{array}{l}0.0341 \\
0.0443\end{array}$ & 0.0272 & $\begin{array}{l}0.03 .54 \\
0.0480\end{array}$ \\
\hline \multirow[t]{2}{*}{$N(x, y)$} & 43 & 41 & 43 & 42 \\
\hline & 46 & 44 & 46 & 40 \\
\hline$r$ & $\begin{array}{l}0.7107 \\
0.7496\end{array}$ & $\begin{array}{l}0.6976 \\
0.7+17\end{array}$ & $\begin{array}{l}0.6867 \\
0.7264\end{array}$ & $\begin{array}{l}0.5291 \\
0.6856\end{array}$ \\
\hline \multirow[t]{2}{*}{$F<1 \%$} & + & + & + & + \\
\hline & $*$ & + & + & 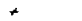 \\
\hline
\end{tabular}

\begin{tabular}{|c|c|c|c|c|}
\hline & Cage 1 & Cage z & Cage $J$ & Cage 4 \\
\hline $\mathrm{a}^{\circ}$ & 4.6220 & 4.7260 & 6.9280 & $7-4700$ \\
\hline b' & $\begin{array}{l}0.1343 \\
0.1581\end{array}$ & $\begin{array}{l}0.1384 \\
0.1801\end{array}$ & $\begin{array}{l}0.0889 \\
0.1464\end{array}$ & $\begin{array}{l}0.1092 \\
0.1826\end{array}$ \\
\hline$N(x, y)$ & $\begin{array}{l}43 \\
40\end{array}$ & $\begin{array}{l}41 \\
44\end{array}$ & $\begin{array}{l}43 \\
46\end{array}$ & $\begin{array}{l}42 \\
46\end{array}$ \\
\hline$r$ & $\begin{array}{l}0.7455 \\
0.7624\end{array}$ & $\begin{array}{l}0.7499 \\
0.7698\end{array}$ & $\begin{array}{l}0.6465 \\
0.7020\end{array}$ & $\begin{array}{l}0.5315 \\
0.6967\end{array}$ \\
\hline$F<1 \%$ & + & + & + & + \\
\hline & + & $*$ & + & + \\
\hline
\end{tabular}

Relation poids-longueur $W=A \times L *$

\begin{tabular}{|c|c|c|c|c|}
\hline & Cage 1 & Cage 2 & Cage 3 & Cage 4 \\
\hline$A \times 10^{-3}$ & 2.3175 & 1.5107 & $4.094 \mathrm{~S}$ & 6.0213 \\
\hline$B$ & $\begin{array}{l}3.0079 \\
3.1990 \\
3.6880\end{array}$ & $\begin{array}{l}0.0368 \\
3.3925 \\
3.708 .5\end{array}$ & $\begin{array}{l}1.0084 \\
3.0550 \\
3.5771\end{array}$ & $\begin{array}{r}1.1781 \\
2.935 \theta \\
3.533 ?\end{array}$ \\
\hline \multirow[t]{2}{*}{$N\{x, y\}$} & 43 & 41 & 43 & 42 \\
\hline & 40 & 44 & 40 & 46 \\
\hline$r$ & $\begin{array}{l}0.8434 \\
0.8746\end{array}$ & $\begin{array}{l}0.9995 \\
0.9451\end{array}$ & $\begin{array}{l}0.8312 \\
0.9475\end{array}$ & $\begin{array}{l}0.908 \mathrm{~B} \\
0.9450\end{array}$ \\
\hline \multirow[t]{2}{*}{$F<1 \%$} & + & + & + & + \\
\hline & + & + & + & + \\
\hline
\end{tabular}

L'analyse statistique des croissances linéaires conduit à l'acceptation de l'hypothèse d'égalité des pentes pour les cages 1,2 et 4 (F $2.8=0,083$ à $5 \%$ ).

La relation entre la longueur totale de l'alevin (Lt) et la largeur de la bouche (lb) suit un modèle linéaire (fig. 2). 

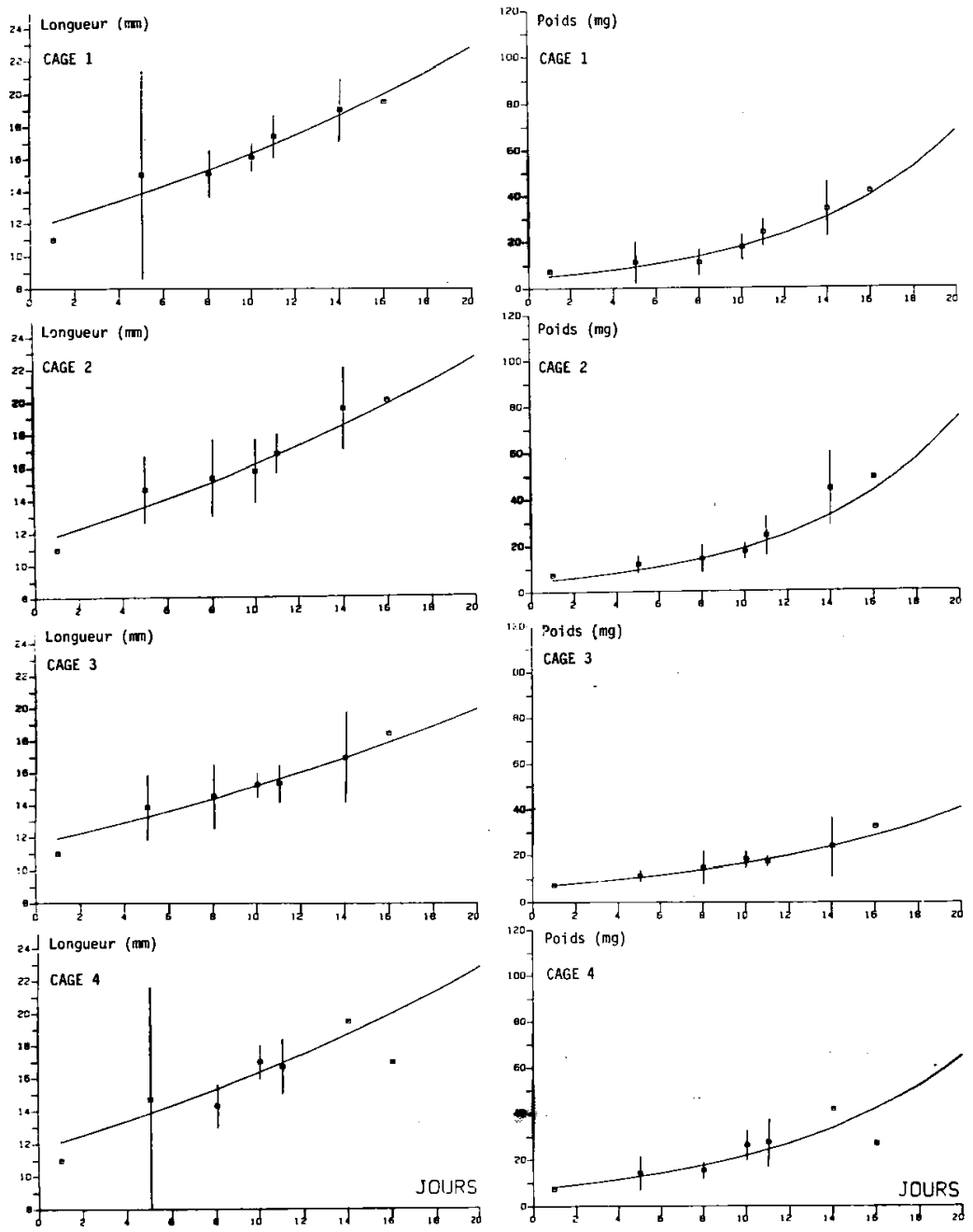

Fig. 1. Courbes de croissance linéaire et pondérale. 


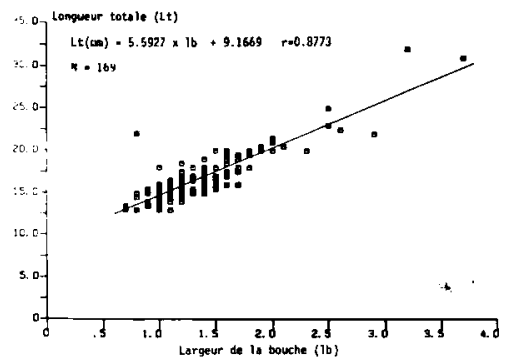

Fig. 2. Relation entre la longueur totale de l'alevin et la largeur de la bouche.

Au cours des 16 jours d'élevage, les brochetons ont grandi de 0.43 à $0.56 \mathrm{~mm} / \mathrm{j}$ (Tableau II). Pour des mises en charges de 1500 brochetons $/ \mathrm{m}^{3}$, Zaugg \& Pedroli (1984) ont observé, en cages illuminées et pendant $40 \mathrm{j}$, un accroissement de $0,75 \mathrm{~mm} / \mathrm{j}$. En etang, la vitesse de croissance est encore plus rapide, Souchon (1980) relève des longueurs moyennes de $95 \mathrm{~mm}$ en 45 jours $(2,1 \mathrm{~mm} / \mathrm{j})$.

Le cuefficient de croissance pondérale est optimal (1.753) pour une ration totale de 17 grammes (Tableau II). Pour des rations 2 fois plus importantes ou 2 fois plus faibles, on observe un abaissement de $23 \%$ et $5 \%$ du coefficient, respectivement. Pour une faible mise en charge et très forte ration (cage 4), le coefficient est voisin de la valeur optimale. A l'issue de 15 jours d'élevage, Jager (1980) observe un coefficien d'environ 2,9, pour des mises en charges de 3000 à 6000 alevins $/ \mathrm{m}^{3}$.

\subsection{Comportement alimentaire des brochetons}

\section{RYTHME DE PRISE ALIMENTAIRE}

Le rythme de prise alimentaire a été étudié du 8 juin, $9 \mathrm{~h}$, au 9 juin, $9 \mathrm{~h}$, à une température moyenne de $11.1^{\circ} \mathrm{C}(+/-0.24)$. Les alevins étaient ágés de 32 jours, ils mesuraient de 15 à $17 \mathrm{~mm}$ et pesaient de 17 à 26 mg (fig. 3).
Tableau II. Parametres de croissance.

$\mathrm{Li}$ : longueur initiale en $\mathrm{mm}$ (valeur réelle).

Lf: longueur au bout de 16 jours (valeur theorique).

$\triangle \mathrm{Lj}$ : accroissement linéaire journalier en $\mathrm{mm} / \mathrm{j}$.

$\Delta$ L\%j: accroissement linéaire journalier en $\%$ de la longueur totale (mm) par jour.

Wi : poids initial en $\mathrm{mg}$ (valeur réelle).

Wf : poids au bout de 16 jours (valeur théorique).

G: coefficient de croissance pondérale.

$\mathrm{Rt}$ : ration totale de plancton par cage (mg).

\begin{tabular}{|c|c|c|c|c|}
\hline $\begin{array}{r}\text { Cages } \\
\text { Farametres }\end{array}$ & 1 & 2 & 3 & 4 \\
\hline 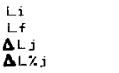 & $\begin{array}{l}11.00 \\
19.85 \\
0.55 \\
5.09\end{array}$ & $\begin{array}{l}11.00 \\
19.75 \\
0.55 \\
4.97\end{array}$ & $\begin{array}{l}11.00 \\
17.93 \\
0.43 \\
3.94\end{array}$ & $\begin{array}{l}11.00 \\
20.00 \\
0.56 \\
5.11\end{array}$ \\
\hline $\begin{array}{l}\text { Wi } \\
\text { Wf } \\
\text { B }\end{array}$ & $\begin{array}{l}7.50 \\
39.64 \\
1.665\end{array}$ & $\begin{array}{l}7.50 \\
43.27 \\
1.753\end{array}$ & $\begin{array}{l}7.50 \\
28.73 \\
1.343\end{array}$ & $\begin{array}{l}7.50 \\
42.19 \\
1.727\end{array}$ \\
\hline Rt & 9019 & 17042 & 33048 & 62776 \\
\hline
\end{tabular}

Les alevins ont généralement un rythme de prise alimentaire "biphasique " : un pic diurne et un pic nocturne.

Les alevins, soumis à une faible ration (cage 1), se nourrissent préférentiellement le jour $(15 \mathrm{~h})$.

Les alevins, ayant reçu de plus grandes rations (cages 2 et 3), ont 2 pics d'alimentation : l'un crépusculaire (18 h), l'autre nocturne ( $3 \mathrm{~h}$ ou $24 \mathrm{~h}$ ).

Les alevins, élevés à faible densité et sous très forte ration (cage 4), n'ont pas de rythme de prise alimentaire marqué.

Le rythme alimentaire est donc influencé par la taille de la ration. Certains auteurs pensent que l'augmentation de la densité des proies favorise la fréquence des contacts, leur répartition en agrégats et la vitesse de consommation des prédateurs (Ivlev 1961, Ware 1972). L'effet de groupe a une importance sur le rythme de prise alimentaire et sur la synchronisation des rythmes individuels (Muller 1976).

L'arythmie observée dans la cage 4 résulterait en partie de la faible densité de poissons. 

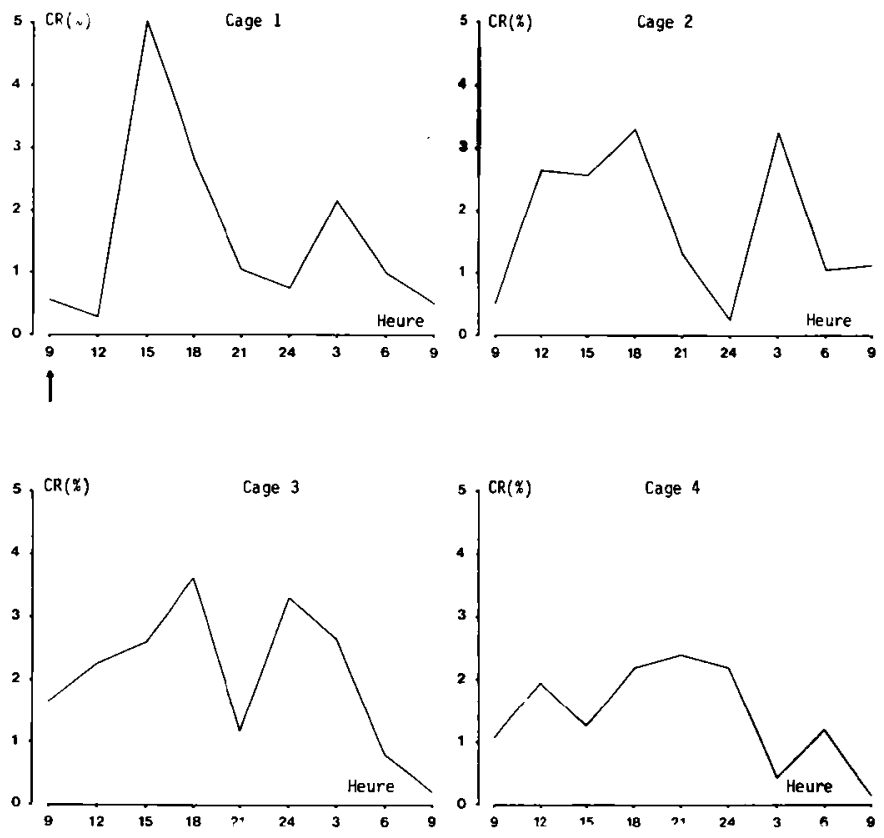

Fig. 3. Rythme de prise alimentaire étudié sur 1 cycle de $24 \mathrm{~h}$. flèche: déversement de plancton.

\section{CANNIBALISME, NOMBRE MOYEN DE PROIES}

Le cannibalisme apparaît dès la taille de $20 \mathrm{~mm}$ et concerne 33 à $50 \%$ des individus selon les cages (Tableau III). C'est à partir de cette mème taille que Hunt \& Carbine (1950), constatent le déclenchement du phénomène. Pour Rahn (1972), il apparaît à $23 \mathrm{~mm}$.

Le nombre moyen de proies ingérées par alevin (exception faite des poissgns à jeun et cannibales) ne varie pas de manière significative selon les 4 rations mais il augmente avec la taille de l'alevin (P 0.025 ; Tahleau IV) Carbine \& Hunt (1950), pour des alevins de 21 à $25 \mathrm{~mm}$, observent un nombre maximal de 26 proies planctoniques par estomac.

\section{REgIME ALIMENTAIRE}

Bosmina longirostris représente 51 à $80 \%$ des proies ingérées (fig. 4). Elle est présente dans au moins $\mathbf{8 0} \%$ des tubes digestifs cxaminés. Daphnia longispina ne représentant au maximum que 12,7\% du régime, peut être présent chez $40 \%$ des alevins examinés.

Les autres Cladocères (Ceriodaphnia serrulata, Holopedium gibbosum ou Sida cristallina) sont rares dans les tubes digestifs. 
Tableau III : Cannibalisme et nombre moyen de proies. $\mathrm{N}$ alv. : nombre d'alevins examinés

$N$ vid. : nombre d'alevins au tube digestif vide

$N$ can. : nombre d'alevins cannibales

$\%$ can. : taux de cannibalisme

$N$ mov p.: nombre moyen de proies

\begin{tabular}{|c|c|c|c|c|c|}
\hline $\begin{array}{c}\text { Cagez } \\
\text { Cl antess }\end{array}$ & $(\operatorname{mm})$ & 2 & 2 & 3 & 4 \\
\hline $12-16$ & 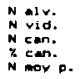 & $\begin{array}{r}14 \\
3 \\
0 \\
0 \\
23\end{array}$ & $\begin{array}{r}16 \\
1 \\
0 \\
0 \\
21\end{array}$ & $\begin{array}{r}28 \\
2 \\
0 \\
0 \\
22\end{array}$ & $\begin{array}{r}21 \\
1 \\
0 \\
0 \\
25\end{array}$ \\
\hline $16-20$ & $\begin{array}{l}N \text { alv. } \\
N \text { rid. } \\
N \text { can. } \\
x \text { can. } \\
N \text { mor } p .\end{array}$ & $\begin{array}{r}25 \\
0 \\
0 \\
0 \\
36\end{array}$ & $\begin{array}{r}19 \\
0 \\
0 \\
0 \\
29\end{array}$ & $\begin{array}{r}14 \\
1 \\
0 \\
0 \\
54\end{array}$ & $\begin{array}{r}12 \\
0 \\
0 \\
0 \\
36\end{array}$ \\
\hline $20-24$ & $\begin{array}{l}N \text { alv. } \\
N \text { vid. } \\
N \text { can. } \\
Z \text { Can. } \\
\text { N tor p. }\end{array}$ & $\begin{array}{r}6 \\
0 \\
3 \\
50 \\
44\end{array}$ & $\begin{array}{r}8 \\
0 \\
3 \\
38 \\
00\end{array}$ & $\begin{array}{l}0 \\
\overline{-} \\
-\end{array}$ & $\begin{array}{r}12 \\
0 \\
4 \\
35 \\
69\end{array}$ \\
\hline
\end{tabular}

Tableau IV : Composition du plancton distribué le 8 juin 1984 (catégories, classes de taille en microns).

\begin{tabular}{|c|c|c|c|c|c|c|}
\hline $\mathrm{Cl} \cos =\mathrm{s}=(\omega)$ & Total & $<400$ & $\begin{array}{l}400 \\
800\end{array}$ & $\begin{array}{l}000- \\
1200\end{array}$ & $\begin{array}{l}1200- \\
1600\end{array}$ & 21600 \\
\hline \multicolumn{7}{|l|}{ Cotegor i es } \\
\hline \multirow{3}{*}{$\begin{array}{l}\text { Dosmina } \\
\text { Daphnia } \\
\text { Autres: } \\
\text { cladoceres } \\
\text { Cyclogides } \\
\text { Di apt omides }\end{array}$} & $\begin{array}{l}35.4 \\
22.9\end{array}$ & 4.2 & $\begin{array}{r}31.2 \\
4.9\end{array}$ & 14.6 & 1.4 & 2.1 \\
\hline & $\begin{array}{r}6.9 \\
20.9 \\
13.9\end{array}$ & $=$ & $\begin{array}{l}6.9 \\
2.8 \\
-\end{array}$ & $\begin{array}{c}- \\
14.6 \\
0.7\end{array}$ & $\begin{array}{l}-. \\
2.2 \\
9.0\end{array}$ & $\begin{array}{c}- \\
1.4 \\
4.2\end{array}$ \\
\hline & Total & 4.2 & 45.8 & 29.9 & 12.5 & 7.7 \\
\hline
\end{tabular}

Les jeunes stades de Cyclopides et les stades adultes de Diaptomides constituent moins de $30 \%$ des proies mais sont très fréquents dans les tubes digestifs.

L'analyse statistique des données met en évidence 3 points :

- pour une ration donnée, les différences que l'on observe entre les catégories de proies sont significatives ( $P<0.05 \%$ )

- mais les différences entre les classes d'alevins ne le sont pas.

- pour une classe d'alevins donnée, la variable " ration " n'entraîne pas de différences significatives entre les frequences d'occurence.
TAILLE PROIE - TAILLE PREDATEUR

L'analyse de variance des résultats montre que les différences observées entre les Lmoy., Lmax. et Lmin. sont significatives pour chacune des cages $(\mathrm{P}<0.05 \%$, Fig. 5). Quelle que soit la ration distribuée, Lmoy. et Lmax. augmentent avec la taille de l'alevin. Les moyennes obtenues sont plus élevées chez le's alevins de la cage 4 . Lmin. est indépendant de la taille du brocheton et de la ration de plancton. Des relations linéaires entre Lmax., Lmin., Lmoy. de Cyclops bicuspidatus ingérés et les classes de taille de larves de Stizostedion vitreum, ont èté observées par Mathias \& Li (1982).

\section{SÉLECTION DES PROIES}

D'une manière générale, les alevins recherchent activement le genre Bosmina, compris entre 400 et 800 microns. Ils évitent fortement les Diaptomides et les Cyclopides, quelles que soient leur taille, et les autres cladocères (Tableau V).

Néanmoins, les alevins élevés sous faible ration (cage 1) évitent faiblement les jeunes Cyclopides, inférieurs à 1200 microns et les autres cladocères. Les alevins de la cage 2 évitent le genre Daphnia. quel que soit sa taille. Sous condition de forte ration (cage 3), les alevins évitent faiblement les jeunes stades de Cyclopides, de taille inférieure à 800 microns. Les alevins de la cage 4 sont les seuls a rechercher de grosses Daphmia, de taille supéricure à 1600 microns.

L'étude montre que les alevins sont très sélectifs dans leurs choix vis-à-vis de la catégorie de proie et de la taille. Ce choix est fonction des conditions de ration : une faible ration (cage 1 ) pousse l'alevin à diversifier son régime; une forte ration couplée à une faible densité de poissons (cage 4) induit une capture active de grosses proies. En milieu naturel. Mongeau (1955) constate que $88 \%$ des organismes ingérés par des alevins de 20 à $40 \mathrm{~mm}$ sont des Cladocères. L'hypothèsé de sélection vis-à-vis de la taille a été int roduite par Brooks \& Dodson (1965) et plus tard verifiée par plusieurs auteurs (Eggers 1977. Schmidt \& O'Brien 1982, Wright \& O'Brien 1982). Zaret (1975) a démontré que la prédation serait déterminée plus par la coloration du plancton que par la taille. Pour Confer \& Blades (1975) et Janssen (1976), les facteurs déterminants de prédation pour les alevins chasseurs de proies sont : la visibilité des proies, l'acuité visuelle des alevins et la 

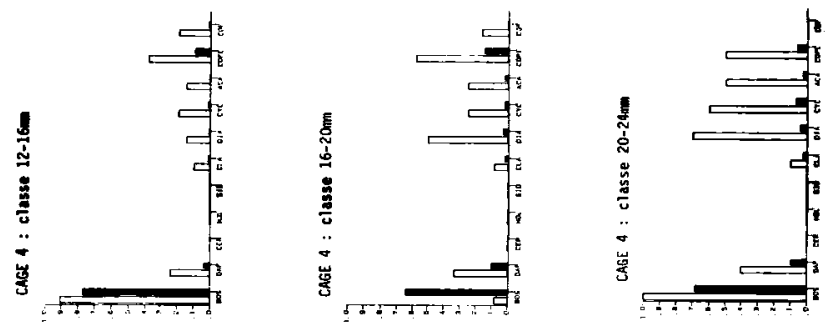

(8)
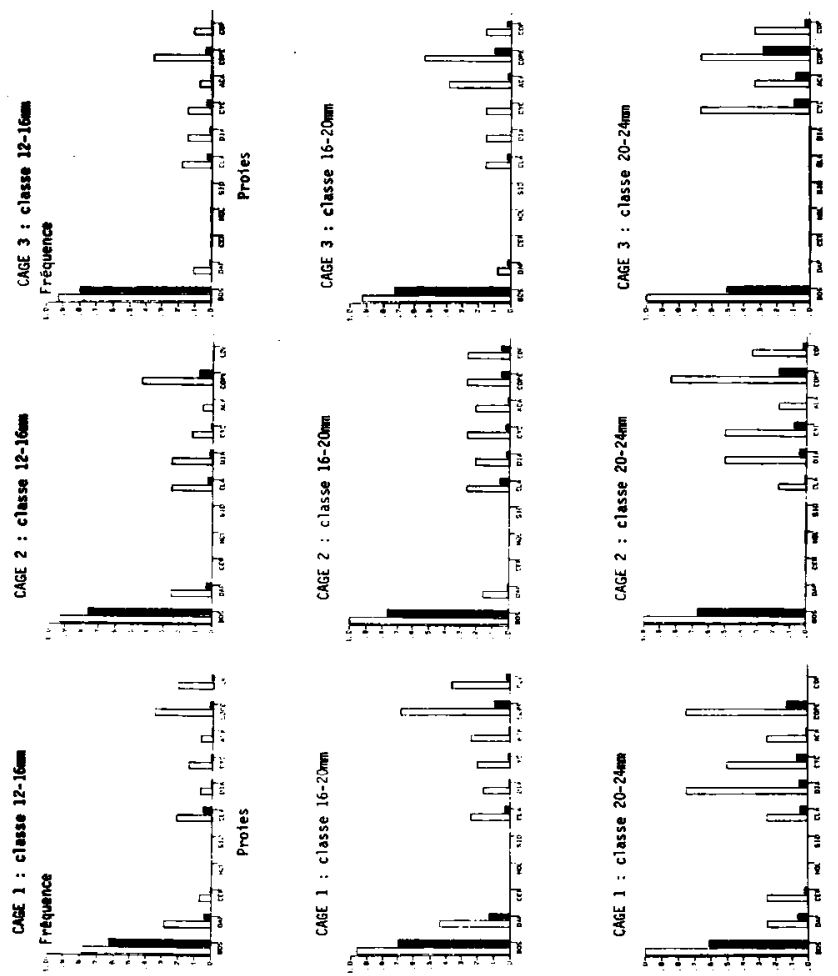

rıg. $4(\mathrm{a}, \mathrm{b})$ : Composition détaillée du régime alimentaire :

En blanc: proportion des tubes digestifs contenant le taxon $i$;

En noir : proportion du taxon i dans les tubes digestifs. BOS : Bosmina longirostris ; DAP : Daphnia longispina : CER : Ceriodaphnia sp. ; HOL : Holopeditum sp.; SID : Sida crystallina; CLA : Cladocères indéterminés:

DIA : Diaptomus cyaneus : CYC : Cyclops vicinus ; ACA : Acanthocyclops sp. : COPE : Copépodites indéterminés: COP : Copépodes indéterminés. 


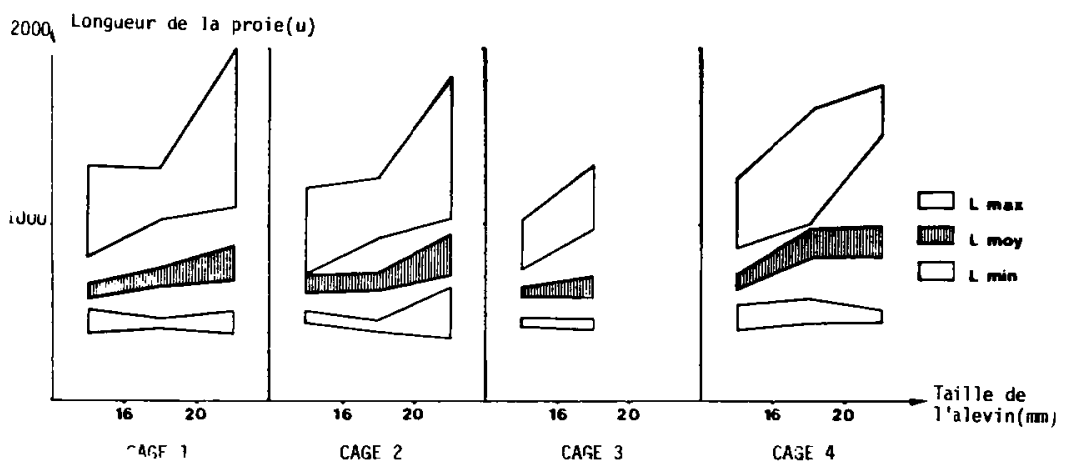

Fig. 5 : Longueurs moyennes des proies ingérées selon la taille de l'alevin.

Tibluau V: Indices d'électivité selon les 5 catégories de proies réparties en classes de taille de $\mathbf{4 0 0}$ microns. Chiffres soulignés: proie activement sélectionnée.

\begin{tabular}{|c|c|c|c|c|c|c|}
\hline $\begin{array}{l}\text { Clasues } \\
\text { Proite: }\end{array}$ & (u) & $<400$ & $\begin{array}{l}400- \\
000\end{array}$ & $\begin{array}{l}900- \\
1200\end{array}$ & $\begin{array}{l}1200- \\
1600\end{array}$ & P160 \\
\hline Bosmina & $\begin{array}{l}1 \\
2 \\
3 \\
4\end{array}$ & $\begin{array}{l}-0.527 \\
-0.714 \\
-0.750 \\
-0.626\end{array}$ & $\frac{+0.364}{+0.426}$ & & & \\
\hline Daphrita & & & $\begin{array}{l}-1 \\
-0.922 \\
-1 \\
-0.556\end{array}$ & $\begin{array}{l}-0.529 \\
-0.706 \\
-0.947 \\
-0.947\end{array}$ & $\begin{array}{r}+0.723 \\
-0.558 \\
+0.151 \\
+0.576\end{array}$ & $\begin{array}{l}-0.31: \\
-1 \\
-1 \\
+0.176\end{array}$ \\
\hline $\begin{array}{l}\text { 7utres } \\
\text { cl adoce }\end{array}$ & eres & . & $\begin{array}{l}-0.221 \\
-0.769 \\
-0.720 \\
-0.917\end{array}$ & & & \\
\hline \multicolumn{2}{|c|}{ Lycl opi des } & & $\begin{array}{r}-0.244 \\
+0.300 \\
-0.697 \\
-0.513\end{array}$ & $\begin{array}{l}-0.292 \\
-0.364 \\
-0.513 \\
-0.339\end{array}$ & $\begin{array}{l}-0.555 \\
-0.024 \\
-0.615 \\
-0.556\end{array}$ & $\begin{array}{l}-0.474 \\
-0.333 \\
-1 \\
-0.556\end{array}$ \\
\hline \multicolumn{3}{|c|}{ ') Laptomi des } & & $\begin{array}{l}-1 \\
-1 \\
-0.556 \\
-1\end{array}$ & $\begin{array}{l}-0.651 \\
-0.513 \\
-0.735 \\
-0.573\end{array}$ & $\begin{array}{l}-0.905 \\
-0.909 \\
-1 \\
-0.905\end{array}$ \\
\hline
\end{tabular}

structure de leur bouche. L'importance de ces facleurs est confirmée par Hessen (1985).

La composition relative d'une communauté zooplanctonique peut refléter les effets de la prédation 'xercés par les poissons (Hrbacek 1962, Lynch \& al. 981. Drenner \& al. 1982, 1984a, 1984b, Vanni 1987.
Lazzaro 1987). Toutefois, il est peu probable que seule la population d'alevins de Brochet modifie la composition du plancton d'un milieu naturel.

\section{Conclusions}

Au cours de cet te expérience, on a pu constater que la croissance est faible en regard de celles ennegistrées en étangs ou en cages illuminées, où la nour riture n'est pas un facteur limitant. Le milieu " cage "favorise le déclenchement précoce du cannibalisme. L'importance de la présence de végétaux et même de la nature de la couverture végétale a souvent été soulignėe, notamment par Hakkari \& Bagge (1985). Ces végétaux offrent un nombre de caches plus important et favorise le développement d'une nourriture diversifiée.

L'expérience a montré qu'il n'est pas nécessaire de déverser de grandes quantités de plancton ; au contraire, cette pratique entraîne des effets inverses à ceux escomptés. Pour 16 jours d'élevage, la ration optimale est de 17 grammes de plancton pour une densité initiale de 600 brochetons par $\mathrm{m}^{3}$.

Pour le cycle étudié, les alevins sélectionnent surtout des proies de taille comprise entre 400 et 800 microns, représentée par Bosmina longirostris au moment de l'étude. 
Deux applications de cet te étudè peuvent être envisagées qui nécessitent la réalisation d'expériences complémentaires:

- modélisation de l'intensité de la prédation sur la communauté zooplanctonique, comme l'ont tenté certains auteurs (Eggers 1977, Wright \& O'Brien 1984. Abrams 1987),

- utilisation de certains résultats obtenus (croissance, ration optimale et calibrage des proies) pour la production d'alevins de repeuplement : Brochet, mais également Perche, Sandre et Gardon.

\section{Travaux cltés}

Abrams (P.). 1987. - Indirect interactions between species that share a predator : varieties of indirect effects. In Kerfoot (W.C.) (ed.). Predation, direct and indirect impacts on aquatic communities : 38-54. University Press of New England.

Applegate (R.L.). 1981. - Food selection of Muskellunge fry. Prog. Fish. Cult., $43: 136-139$.

Beyerle (G.B.) \& Williams (J.E.). 1968 - Some observations of food selectivity by Northern Pike in aquaria. Trans. Am. Fish. Soc., $97: 28-31$.

Boutry (E.). 1983. - Les Corégones du Léman : Pêches, repeuplement et essais d'élevage de juvéniles en cages immergées. Rapport Institut de Limnologie, Thonon-les-bains. I.L. 83/10:32 p.

Brooks (J.L.) \& Dodsun (S.J.). 1965. - Predation, body size and composition of zooplankton. Science, $50: 29.35$.

Bry (C.) \& Gillet (C.). 1980. - Réduction du cannibalisme précoce chez le Brochet (Esox lucius, L.) par isolement des fratries. B.F.P., 277 : 142-153.

Chenoweth (S.B.). 1970. - Seasonal variations in condition of larval Herring in Boothbay of Maine Coast. J. Fish. Res. Bd. Can., 27 (10): $1875 \cdot 1879$.

Chodorowska (W.) \& Chodorowski (A.). 1969. - Les besoins nutritifs des alevins de poissons carnassiers. Verh. Internat. Verein. Limnol., 17 : 1082-1089.

Chodorow'ska (W.) \& Chodorowski (A) 1975. - Substitution d'éléments de la diète chez les alevins de poissons carnassiers. Verh. Intemat. Verein. Limnol, 19:2556-2562.

Confer (J.L.) \& Blades (P.I.). 1975. - Omnivorous zooplankton and planktivorous fish. Limmol. Oceanogr., $20: 571-579$.

Dauba (F.). 1983. - Etude hydrobiologique du lac de Pareloup. Contrat ENSAT/EDF (REAM) : 64 p + annexes.

Drenner (R.W.), Vinyard (G.L.), Guphen (M.) \& Mc Comas (R.). 1982. - Feeding behavior of the Cichlid, Sarotherodon galilaeum, selective predation on Lake Kinneret zooplankton. Hydrobiologia, 87 : 17-20.

Drenner (R.W.), Nummert (J.R.), De Noyelles (F.) \& Kettle (D.). 1984a. - Selective particle ingestion by a filter.feeding fish and its impact on phytoplankton community structure. $\mathrm{Lim}$ nol. Oceonogr., 29 (5): $941-948$.

Drenner (R.W.), Taylor (S.B.), Lazzanc (X.) \& Kettle (D.). 1984b. Particle-grazing and plankton community impact of an omnivorous cichlid. Trans. Am. Fish. Soc., $113: 397.402$

Eggers (D.M.). 1977, - The nature of prey selection by planktivorous fish. Ecology, $58: 46-59$

Fago (D.M.). 1977. - Northem Pike production in managed spawning and rearing marshes. Wisc. Depart. Nat. Res. Tech. Bull, $96: 1.30$.

Gulland (J.A.). 1965. - Survival of the youngest stages of $f$ ish and its relation to year-class strength. Spec. Publ. ICNAF, 6: 363-371.
Hakkari (L.) \& Bagge (P.). [985. - On the fry densilies of pike (Esox lucius, L.) in Lake Saimaa, Finland. Verh. Intermat. Verein. Limnol., $22: 2560.2565$.

Ilessen (D.O.). 1985. - Selective zooplankton predation by preadult roach (Rutilus rutilus, L.): the size-selective hypothesis versus the visibility-selective hypothesis. Hydrobiologia, 24 : 73-79.

Hrbacek (J.). 1962. - Species composition and the amount of zooplankton in relation to the fish stock. Rozpr. Ceskoslov. Akad. Ved., $72(10)$ : $1-116$.

Hunt (B.P.) \& Carbine (W.F.), 1950. - Food of young Pike (Esox lucius, L and associated fishes in Peterson's ditches Hough. ton Lake, Michigan. Trans. Amt. Fisht. Soc., $80: 67-83$.

Ivlev (V.S.) 1961. - Experimental ecology of the feeding of fishes. Yale University Press, New Haven : 302 p.

Jager (T.), 1980. - Aufzucht von Hechisetzlingen in erleuchteten netzgehagen. Fischer und Teichwin. $11:$ 323-326.

Jager (T.). 1982. - Erfahrungs bericht über der verlauf einer privatwirtschaftlichen fischsetzlings produktion im beleucheteten netzgehegen. Osterreichs Fischerei, 36 : 234241.

Janssen (J.t. 1976. - Feeding modes and prey size selection of the alewife (Alosa pseudoharengus). J. Fish. Res. Bd. Can., 33 : 1972-1975.

Lazzaro (X.). 1987. - A review of planktivorous fishes : their evolution feeding behaviours selectivities and impacts. Hydrobio logia, 146: 97-167.

Lynch (M) \& Shapiro (J.). 1981. -- Predation, enrichment and phytoplankton community structure. Limnol. Oceanogr., 26 : 86-102.

Mathias (J A.) \& Li (S.). 1982. - Feeding habits of walleye larvae and juvenile : comparative laboratory and field studies. Trans. Am. Fish. Suc., $111: 722.735$.

Mongeau (J.R.). 1955. - Comportement alimentaire du Brochet commun, Esox lucius L. dans deux lacs du Parc du MontTremblant, Province du Ouebec. Th. Maitrise es Sciences, Univ. Montreal, Faculté des Sciences: 137 p.

Rahn (I.). 1972. - Produktion in intensivanlagen. Z. Bimentisherei D.D.R., $19: 124 \cdot 127$

Saksena (V.) \& Houde (E.D.). 1972. - Effect of food level on the growth and survival of laboratory reared larvae of Bay Anchovy (Anchoa mitchillt, V.) and Scaled Sardine (Harengtala pensacolae, G.). J. Exp: Mar. Biol. Ecol., $8: 249.258$

Souchon (Y.). 1980. - Effet de la densite initiale de petiplement sur la survie et la croissance du Brochel (Esox lucius, L.) éleve jusquicu stade de brocheton (45 jours). In Billard (R.) (ed.) La Pisciculture en étang. INRA Publ. Paris. : $309-316$.

Schmidt (D.R.) \& O'Brien (W.J.). 1982. - Planktivorous feeding ecology of Artic Grayling (Thymallus arricus). Can. J. Fish. Aquat. Sci, 39:375-482.

Vanni (M.J.). 1986. - Fish predation and zooplankton demo graphy : indirect effects. Ecology, $67: 337-354$.

Wright (DI.) \& O'Brien (WJ.). 1982 - Differential location of Chaoborus larvae and Daphnia by fish : the importance of motion on visible size. Am. Mid. Natur, 108 (1) : 68-73.

Wright (D.1.)\& O'Brien (W.J.). 1984. - The development and field test of a tactical model of the planktivorous feeding of White Crappie (Poxomis annularis). Ecol. Monogr, 54 : 65-98.

Zarel (J.M.) 1975. - Fish predation on Bosmina longinosiris: body-size selection versus visibility selection. Ecology, 56 : 232-237.

Zaugg (B.) \& Pedroli (J.C.). 1984 - Elevage de jeunes poissons en cages éclairées, inmergées dans le milieu naturel : expérimentalion d'une pisciculture flottante sur le lac de Neuchátel. Les Cahiers de la Pèche, 43 : 53-87. Office Fédèral de la Protection de I'Environnement, Berne. 